\title{
Competing ferromagnetic and nematic alignment in self-propelled polar particles
}

\author{
Sandrine Ngo, ${ }^{1,2}$ Francesco Ginelli, ${ }^{3,4}$ and Hugues Chaté ${ }^{1,2}$ \\ ${ }^{1}$ Service de Physique de l'État Condensé, CEA-Saclay, 91191 Gif-sur-Yvette, France \\ ${ }^{2}$ Max-Planck-Institute for the Physics of Complex Systems, \\ Nöthnitzer Straße 38, D-01187 Dresden, Germany \\ ${ }^{3}$ Istituto dei Sistemi Complessi, CNR, via dei Taurini 19, I-00185 Roma, Italy \\ ${ }^{4}$ SUPA, Institute for Complex Systems and Mathematical Biology, \\ King's College, University of Aberdeen, Aberdeen AB24 3UE, United Kingdom
}

(Dated: November 24, 2018)

\begin{abstract}
We study a Vicsek-style model of self-propelled particles where ferromagnetic and nematic alignment compete in both the usual "metric" version and in the "metric-free" case where a particle interacts with its Voronoi neighbors. We show that the phase diagram of this out-of-equilibrium XY model is similar to that of its equilibrium counterpart: the properties of the fully-nematic model, studied before in [8], are thus robust to the introduction of a modest bias of interactions towards ferromagnetic alignment. The direct transitions between polar and nematic ordered phases are shown to be discontinuous in the metric case, and continuous, belonging to the Ising universality class, in the metric-free version.
\end{abstract}

PACS numbers: 05.65.+b, 87.18.Gh, 64.60.De

Collective motion is a topic currently enjoying interest in various communities [1-4]. Within (statistical) physics, the seminal work of Vicsek et al. 5], followed by the remarkable calculation of Toner and Tu [6], has offered to view the emergence of collective motion in leaderless groups of identical individuals as the spontaneous breaking of rotational invariance. The celebrated Vicsek model, which consists of self-propelled particles aligning ferromagnetically their orientations with that of their neighbors in the presence of noise, was originally presented - and rightly so - as an out-of-equilibrium XY model where spins are forced to move. As is now wellknown, the Vicsek model is endowed with properties very different from those of the XY model: in two dimensions, true long-range polar (orientational) order emerges [6] from a discontinuous phase transition [7], and the longrange correlations and anomalous fluctuations predicted by Toner and $\mathrm{Tu}$ for the ordered collective motion phase, although not observed in the region near onset, are indeed present in a large portion of parameter space.

Other, Vicsek-style, flocking models have been introduced which serve as key members of different universality classes for "dry active matter" [2], i.e. situations in which global momentum is not conserved and hydrodynamic interactions play no significant role. A prominent case is the "self-propelled rods" model, in which the ferromagnetic interaction of the Vicsek model is replaced by nematic alignment [8], in line with the typical outcome of inelastic collisions between moving elongated objects. Switching from ferromagnetic to nematic symmetry of interactions in this other out-of-equilibrium XY model, changes the symmetry of the ordered phase (which is then nematic), in line with the symmetry change of the quasi-ordered phase of the corresponding equilibrium version [9]. Numerical results [8] suggest that like the original (ferromagnetic) Vicsek model, the nematic order ob- (a)

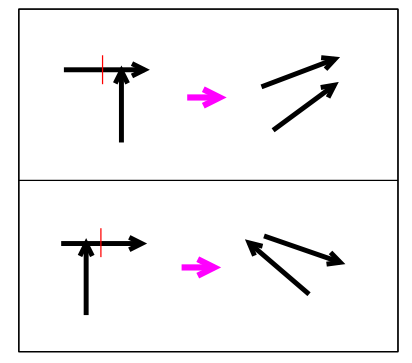

(b)

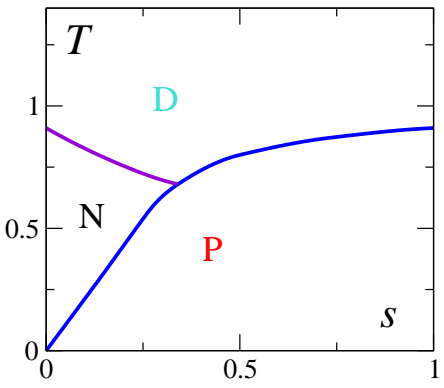

FIG. 1: (color online) (a) Sketch of two self-propelled needlelike objects (black arrows) moving at the same speed in some overdamped dynamics, which collide with an incoming angle of exactly $\frac{\pi}{2}$. The middle of each rod is indicated by the thin red line. If the impact point is along the first half of the hit needle, (some degree of) polar alignment is expected (top panel), whereas anti-alignment will typically occur for an impact point at the rear (bottom panel). Friction forces may very well, though, lead to polar alignment, not anti-alignment, even if the impact point is slightly beyond the first half of the needle. In such a case, the nematic symmetry of interactions would be weakly broken and biased towards ferromagnetic alignment. (b) Schematic phase diagram of an equilibrium generalized XY model with Hamiltonian $H=-\sum_{\langle i j\rangle} s \cos \left[\theta_{i}-\theta_{j}\right]+(1-s) \cos \left[2\left(\theta_{i}-\theta_{j}\right)\right]($ after [9]). Interactions are purely nematic (resp. ferromagnetic) for $s=0$ (resp. 1). D, P, and N, respectively stand for disorder, polar order, and nematic order.

served is truly long-range, but no Toner-Tu-like calculation is available to confirm this at some analytical level.

One might object that the properties of the "nematic Vicsek model" are not robust in the sense that the strict nematic symmetry of its interactions may be generically broken, albeit weakly, by "friction" effects during collisions between actual rods (Fig. 1a). On the other hand, 
in equilibrium, it is known that the quasi-ordered phase of the XY model with nematic interactions resists a modest amount of ferromagnetic alignment (Fig. 10) [9].

In this Rapid Communication, we introduce and study an out-of-equilibrium, Vicsek-style, version of such a generalized XY model where ferromagnetic and nematic alignment compete. We show that its phase diagram is similar to that of its equilibrium counterpart, although rendered complicated by density-segregated sub-phases. Thus, the fully-nematic Vicsek model is robust to the introduction of a modest bias of interactions towards ferromagnetic alignment, alleviating the concern raised above for collisions of actual rods. We also show that this conclusion holds in the case of "topological" neighbors where interactions are not limited to some metric zone but occur with those objects defining the first shell of Voronoi cells around the considered particle. In both the metric and this "metric-free" case, we study the direct polar-nematic transition present in phase diagrams like that of Fig. 10. We provide evidence that it seems to be discontinuous in the metric model, but continuous in the metric-free case, with critical exponents of the Ising universality class, as in equilibrium [9]. We finally discuss the inherent difficulties in deriving hydrodynamic theories in the case of mixed ferromagnetic and nematic interactions.

Our starting point is a Vicsek-style model with competing ferromagnetic and nematic interactions: $N$ point particles move off-lattice at constant speed $v_{0}$ on a two dimensional $L \times L$ torus; particle $j$ is defined by its position $\mathbf{r}_{j}^{t}$ and orientation $\theta_{j}^{t}$, updated according to

$$
\begin{aligned}
\theta_{j}^{t+1} & =\arg \left[\sum_{k \sim j} g_{s}\left(\theta_{j}^{t}, \theta_{k}^{t}\right)\right]+\eta \xi_{j}^{t} \\
\mathbf{r}_{j}^{t+1} & =\mathbf{r}_{j}^{t}+v_{0} \mathbf{v}_{j}^{t+1}
\end{aligned}
$$

where $\mathbf{v}_{j}^{t}=\left(\cos \theta_{j}^{t}, \sin \theta_{j}^{t}\right)^{T}$, the sum is taken over all particles $k$ within unit distance from particle $j$ (including $j$ itself), $\xi$ is a white noise uniformly distributed in $\left[-\frac{\pi}{2}, \frac{\pi}{2}\right]$, and the complex stochastic function $g_{s}\left(\theta, \theta^{\prime}\right)$ is:

$$
g_{s}\left(\theta, \theta^{\prime}\right)= \begin{cases}e^{i \theta^{\prime}} & \text { with prob. } s \\ \operatorname{sign}\left[\cos \left(\theta-\theta^{\prime}\right)\right] e^{i \theta^{\prime}} & \text { otherwise }\end{cases}
$$

Note that in the second case $g_{s}\left(\theta, \theta^{\prime}\right)$ is invariant under the transformation $\theta^{\prime} \rightarrow \theta^{\prime}+\pi$ and thus codes nematic alignment, while the first case expresses ferromagnetic interaction. For $s=0$, this model reduces to model studied in [8], while $s=1$ is fully equivalent to the standard Vicsek model. Thus, $s$ is a key parameter governing the relative weight of ferromagnetic interactions which comes in addition to the two main parameters, the density $\rho=N / L^{2}$ and the noise strength $\eta$. In the following, we focus on a low density system at $\rho=\frac{1}{8}$ with $v_{0}=\frac{1}{2}$, as in [8], and study the $(s, \eta)$ parameter plane. [10]

Systematic scans were performed for different sizes. While $L=512$ allows for a rough determination of the

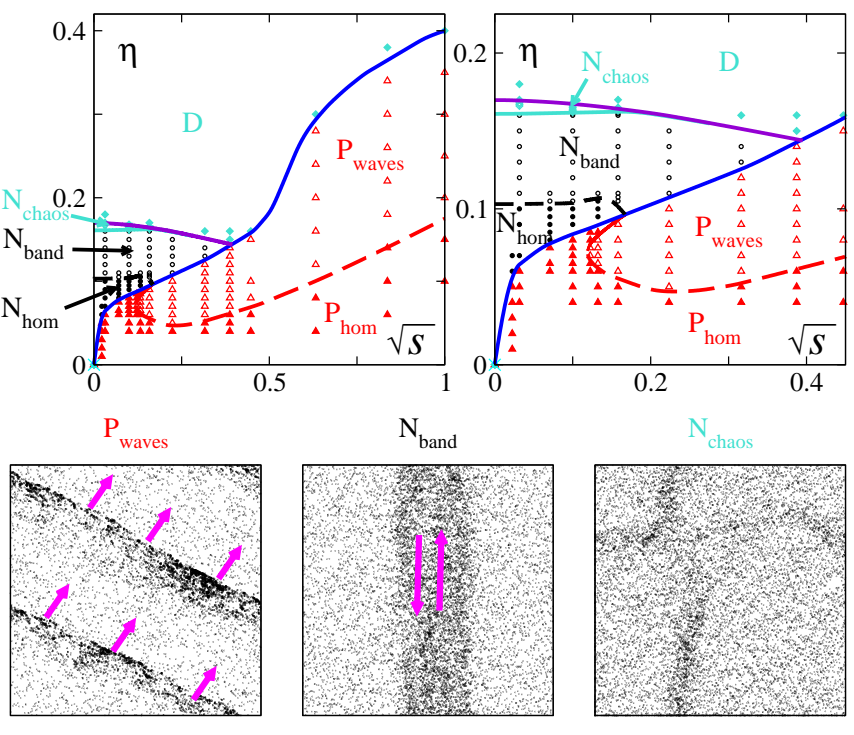

FIG. 2: (color online) Top panels: Phase diagram of the generalized Vicsek model with competing ferromagnetic and nematic interactions $\left(\rho=\frac{1}{8}, L=1024, v_{0}=\frac{1}{2}\right)$ (the top right panel is a close up of the left panel.). Results are presented in function of $\sqrt{s}$ for clarity. Symbols located where individual runs were performed, coding the resulting observed phase: red triangles for polar order, black circles for nematic order, and cyan diamonds for disorder. Full/empty symbols code for homogeneous/density segregated phases. Bottom panels: typical snapshots of the 3 density segregated phases present (system size $L=1024$ ) Left: $\mathrm{P}_{\text {waves }}$ at $s=0.05, \eta=0.1$. Middle: $\mathrm{N}_{\text {band }}$ at $s=0.01, \eta=0.14$. Right: $\mathrm{N}_{\text {chaos }}$ at $s=0.01$, $\eta=0.166$. The thick magenta arrows indicate the direction of motion of particles.

main features of the phase diagram, one needs larger sizes to capture its details, mostly because the segregated banded states of the fully nematic $(s=0)$ model arise clearly only for large systems [8]. Polar and nematic order were characterized by means of the two time-dependent global scalar order parameters $P(t)=\left|\left\langle\exp \left(i \theta_{j}^{t}\right)\right\rangle_{j}\right|$ (polar) and $Q(t)=\left|\left\langle\exp \left(i 2 \theta_{j}^{t}\right)\right\rangle_{j}\right|$ (nematic), as well as their asymptotic time averages $P=\langle P(t)\rangle_{t}$ and $Q=\langle Q(t)\rangle_{t}$. To detect the various density-segregated phases, we relied on snapshots and movies of coarse-grained density and order parameter fields.

The phase diagram is presented in Fig. 2 for $L=1024$, a size beyond which its various features do not seem to change. Close to the $s=0$ axis, one observes that the various nematic sub-phases described in [8] for the purely nematic case are extended to finite but small $s$ values: the spatially-homogeneous nematically-ordered phase (denoted $\mathrm{N}_{\text {hom }}$ ) can be observed up to $\sqrt{s} \approx 0.17$ for $\eta \approx 0.1$. The segregated phase with a unique, dense, nematically-ordered band occupying a finite fraction of space $\left(\mathrm{N}_{\text {band }}\right.$ ) extends up to $\sqrt{s} \approx 0.4$ for $\eta \approx 0.13$. The spectacular spatiotemporal chaos regime in which thin unstable dense bands elongate, twist, break, collide and form again (denoted $\mathrm{N}_{\text {chaos }}$ ) is limited to a narrow tongue 
near $\eta=0.16$. As $s$ is increased, the width of this tongue decreases, making it difficult to locate numerically; our results suggest, though, that it extends all the way to the meeting point with the polar order region.

Polar order can be observed at arbitrary small $s$, provided $\eta$ is small enough; it is delimited by a smooth line (like in the equilibrium case, see Fig. 2) starting at the origin and ending at $\eta \simeq 0.4$ for $s=1$, the transition point of the Vicsek model at this density[11]. The polar order region is itself divided in two, as expected from our knowledge of the Vicsek model: in a large, tongue-like region bordering the onset of polar order $\left(\mathrm{P}_{\text {waves }}\right.$ in Fig. 2), the ordered phase consists of trains of solitary traveling waves, and is thus different from the Toner-Tu homogeneous phase $\left(\mathrm{P}_{\text {hom }}\right)$ present at lower noise strength.

The phase diagram of our out-of-equilibrium, Vicsekstyle, generalized XY model thus possesses the same general structure as its equilibrium counterpart: a small nematic triangular region is present on the small- $s$ side above a continuous line delimiting polar order. This general structure is complicated by the presence of the various density-segregated ordered subphases. At our numerical resolution the line dividing the polar region (red dashed line in Fig. 2) and that dividing the nematic region (black dashed line) seem to meet the border of the polar region (blue solid line) at the same point [12]. There are thus two different ways of transitioning directly from polar to nematic order, as opposed to only one at equilibrium: at small $s$ values, the P-N transition occurs between spatially-homogeneous phases, while at intermediate $s$ values, it links the two density segregated phases $\mathrm{P}_{\text {waves }}$ and $\mathrm{N}_{\text {band }}$ in Fig. 2 ,

We now turn our attention to the nature of the main transitions (disorder/nematic, disorder/polar, and polar/nematic). Let us first recall that the ordered phases observed all seem numerically to show true long-range order, even the nematic phases $\mathrm{N}_{\text {hom }}$ and $\mathrm{N}_{\text {band }}[8]$. In these last cases, however, as argued in [8], one cannot exclude the possibility that nematic order is only quasilong-range at very large scales. In the following, we assume true long-range order, in agreement with the numerical results.

The transition from disorder to nematic order actually occurs between the chaotic $\mathrm{N}_{\text {chaos }}$ phase and the ordered, segregated $\mathrm{N}_{\text {band }}$ phase. As reported in [8], it is determined by the long-wavelength instability of the band, which leads to the $\mathrm{N}_{\text {chaos }}$ phase. This phase being disordered, albeit with very large intrinsic scales, the nematic order parameter $Q$ shows a discontinuous jump at the transition. The disorder/polar transition occurs between the microscopically disordered phase $\mathrm{D}$ and the segregated phase $\mathrm{P}_{\text {waves }}$. Like for the original Vicsek model, it is also discontinuous, as the polar order parameter takes finite, order 1 values as soon as the waves appear 7].

We studied the two different P-N transitions present. The transition between the segregated phases $\mathrm{P}_{\text {waves }}$ and (a)

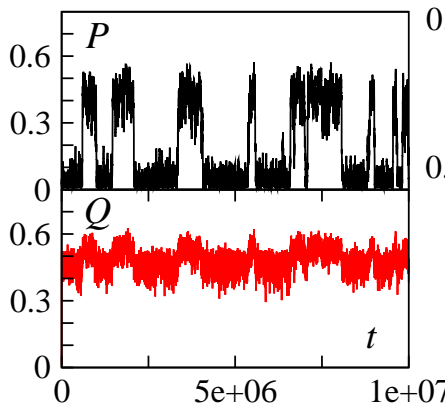

(c)

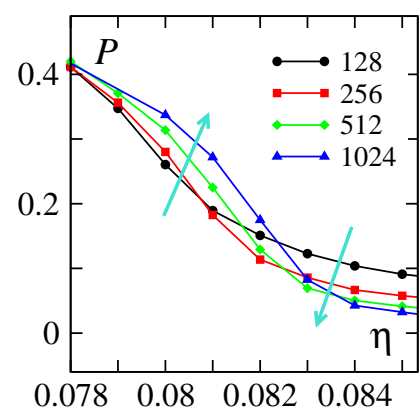

(b)

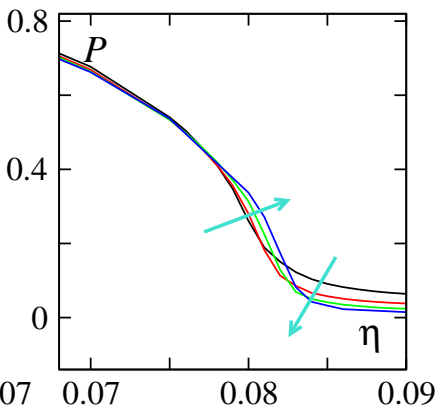

(d)

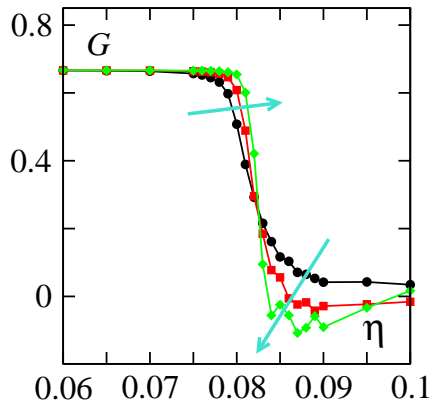

FIG. 3: (color online) Direct transitions from nematic to polar order in the generalized Vicsek model. (a) transition between the segregated phases $\mathrm{P}_{\text {waves }}$ and $\mathrm{N}_{\text {band }}$ at $s=0.1$ occurring near $\eta=0.132$ (time series of polar (top) and nematic (bottom) order parameter for $L=256$ ). (b-d) transition between the homogenous phases $\mathrm{P}_{\text {hom }}$ and $\mathrm{N}_{\text {hom }}$ at $s=0.01$ occurring near $\eta=0.085$. (b,c): order parameter curves at sizes $L=128,256,512,1024$. Panel (c) is a close-up of panel (b). (d): Binder cumulant curves at the same system sizes. The arrows indicate increasing system size.

$\mathrm{N}_{\text {bands }}$ was studied as a function of $\eta$ at $s=0.1$. It is clearly discontinuous, as seen, e.g., in the characteristic flip-flop dynamics of the polar order parameter $P$ in the transition region, leading to a bimodal distribution testifying of phase coexistence (Fig. 3a). The transition between the homogeneous nematic and polar phases is more difficult to characterize. We performed a finite-size scaling study at $s=0.01$, varying $\eta$ around the transition point. The behavior of the $P(\eta)$ curves (Fig. 3b) indicates the premises of a discontinuous transition: although no discontinuity proper is present even at the largest size considered, these curves cross each other, suggesting that a jump may appear at still larger sizes. This conclusion is also borne out of the behavior of the Binder cumulant $G=1-\left\langle P(t)^{4}\right\rangle_{t} /\left(3\left\langle P(t)^{2}\right\rangle_{t}^{2}\right)$, which develops a deeper minimum as the system size is increased (Fig. [3d) [13].

We now report on the properties of the metric-free, "topological" version of our generalized Vicsek model with competing ferromagnetic and nematic alignment. This case is of theoretical interest because no densitysegregated phases are present in the purely ferromagnetic or nematic cases [17 19], opening the way to con- 
tinuous phase transitions (see below). It is also relevant in the context of collective motion. Common sense and some experimental/observational evidence indicate that in groups of higher organisms moving collectively (bird flocks, fish schools, crowds, etc.), one individual, rather than interacting with neighbors located within a given metric zone around itself, takes into account those individuals forming some "angular landscape" to perform navigation decisions [14]. It was suggested for instance that starlings interact with their 7-8 nearest neighbors, irrespective of the flock density [15]. Careful study of fish trajectory data showed that in some schools the stimulus/response function of fish can be modeled by interactions with their first Voronoi neighbors (those whose polygons, in a Voronoi tessellation of space, form the first shell around the focal fish) [16]. Here, following a study of a metric-free version of the Vicsek model [17], we consider these Voronoi neighbors, and we use "vectorial" noise, which means that Eq.(1) is replaced by $\theta_{j}^{t+1}=\arg \left[\sum_{k \sim j} g_{s}\left(\theta_{j}^{t}, \theta_{k}^{t}\right)+\eta \mathcal{N}_{j} \vec{\xi}_{j}^{t}\right]$ where $\vec{\xi}$ is a randomly oriented unit vector and $\mathcal{N}_{j}$ is the current number of (Voronoi) neighbors of particle $j$.

As shown in [17, 18], global density drops out of the problem in metric-free models (it can be scaled out). In practice we worked at unit density, with $v_{0}=0.5$. The phase diagram of this metric-free version of our generalized Vicsek model is shown in Fig. 4a for $L=128$ (diagrams obtained at larger sizes are nearly indistinguishable). As expected, no density-segregated phases are present, leaving a diagram qualitatively similar to that of the equilibrium case. The P-D and N-D transitions are now continuous, as expected from [17, 18] where the $s=1$ and $s=0$ cases were studied. A detailed study of the associated critical exponents will be presented elsewhere. (For the P-D transition, they are consistent with the values reported in [17].) We studied the direct $\mathrm{P}-\mathrm{N}$ transition at $s=0.05$ by finite-size scaling. The (polar) order parameter curves now do not cross each other (Fig. 4b), and the Binder cumulant curves show a minimum which, after deepening at small sizes, eventually start receding at the largest sizes we could probe (Fig. 4c). These qualitative facts point to a continuous transition. Quantitatively, our estimates of critical exponents are as follows: The crossings of the Binder cumulant curves (near $G \sim \frac{2}{3}$, not visible on Fig. 4c) converge to an asymptotic threshold $\eta_{\mathrm{c}}=0.4530(1)$ with an exponent $1 / \nu=1.00(5)$. The location of the maxima of the susceptibility also converge to the same estimated threshold with the same estimated $1 / \nu$, in excellent agreement with the Ising value $\nu=1$ (Fig. $4 \mathrm{~d}$ ). The peak values of the susceptibility diverge with system size with exponent $\gamma / \nu=1.74(2)$, the Ising value being $\frac{7}{4}$ (not shown). The order parameter decreases algebraically at the estimated critical point with exponent $\beta / \nu=0.126(3)$, in close agreement with the Ising value $\frac{1}{8}$ (Fig. 4e) . Thus, (a)

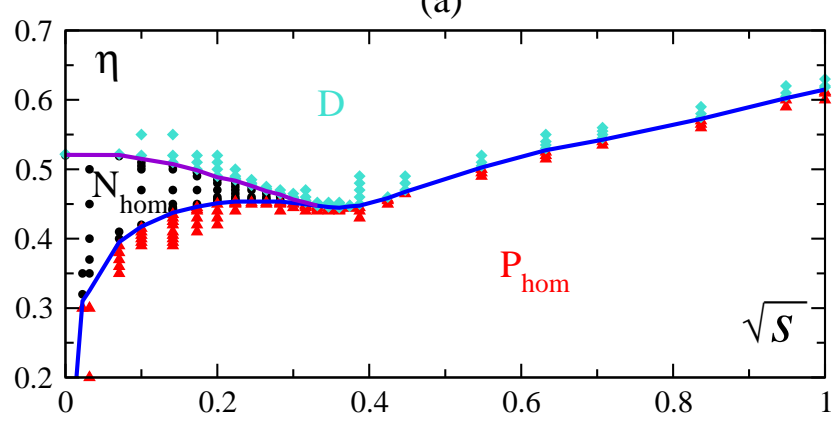

(b)

(c)

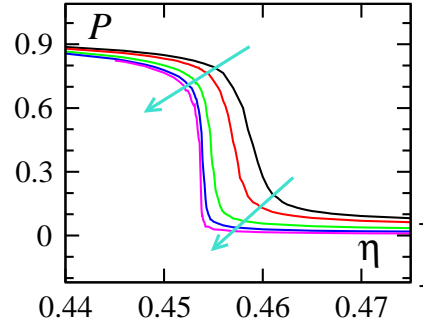

(d)

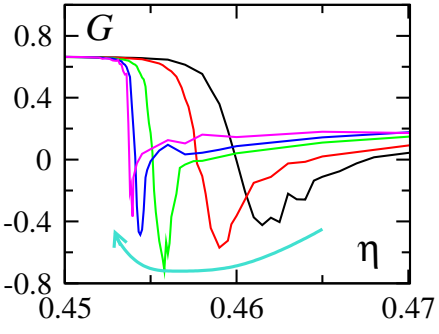

(e)
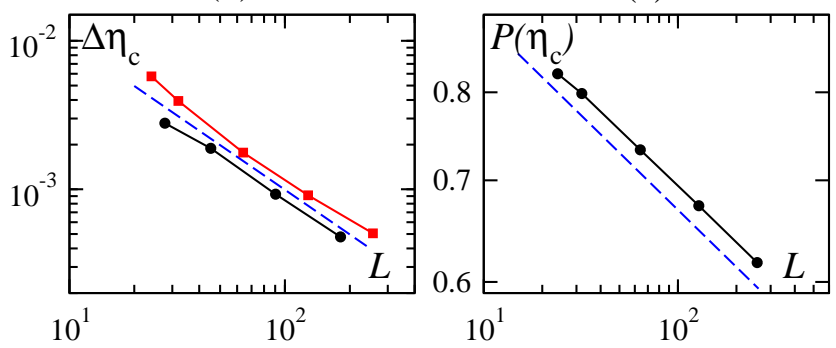

FIG. 4: (color online) Metric-free version of the model where particles interact with their Voronoi neighbors. (a): phase diagram obtained for $L=128$. Legends as in Fig. 2 $\mathrm{bb}$. (bd): finite-size scaling study of the P-N transition occuring at $s=0.05$. (b) and (c): $P(\eta)$ and $G(\eta)$ for $L=24,32,64,128$, and 256 (the arrows indicate increasing system size). The order parameter curves do not cross each other; the Binder cumulant curves show a minimum which first deepens then start receding as $L$ increases. These curves do cross each other when $G \sim \frac{2}{3}$ (not shown). (d): scaling of finite-size distance to asymptotic threshold $\Delta \eta_{\mathrm{c}}(L)=\left|\eta_{c}(L)-\eta_{c}^{\infty}\right|$ for estimated threshold $\eta_{c}^{\infty}=0.4530(1)$. Red squares: $\eta_{\mathrm{c}}(L)$ is the location fo the maximum of susceptibility at size $L$. Black circles: $\eta_{\mathrm{c}}(L)$ is the crossing point of two $G(\eta)$ curves at sizes $L_{1}$ and $L_{2}$ with $L=\sqrt{L_{1} L_{2}}$. The dashed blue line has slope -1. (e): scaling of order parameter at $\eta=\eta_{\mathrm{c}}^{\infty}$. The dashed blue line has slope $-\frac{1}{8}$.

in spite of rather strong finite-size effects as testified by the behavior of the Binder cumulant curves on the nematic side, all estimated critical exponents are very close to their Ising universality class values, as expected from studies of the equilibrium generalized XY model [9].

Before summarizing, we discuss briefly the derivation of a continuous theory describing active matter systems with competing ferromagnetic and nematic alignment interactions. Such a theory must a priori be in terms of a 
polarity field $\mathbf{P}$ and a nematic tensorial field $\mathbf{Q}$ if it is to account for nematically-ordered phases. Baskaran and Marchetti have proposed rather complicated such equations for the case of self-propelled rods interacting via steric exclusion [20]. We have followed the somewhat simpler route of the "Boltzmann" approach used in [18, 21], which is particularly adapted to dilute Vicsek-like models, for the case of competing ferromagnetic and nematic alignment 22]. While details will be published elsewhere [23], we only mention here that the obtained equations for $\mathbf{P}$ and $\mathbf{Q}$ fail to account for the phase diagrams presented here. They confirm the existence of a nematic phase at finite $s$, but are not suitable to describe the polar phase, probably since the truncation scheme used is only valid near onset of nematic order.

In conclusion, we have studied a Vicsek-style model with competing ferromagnetic and nematic alignment in both a metric and a "metric-free" version where interactions take place with Voronoi neighbors. We have shown that the fully nematic case of this out-of-equilibrium XY model ("self-propelled rods") resists some bias toward ferromagnetic alignment, thus conferring some robustness to its nematically-ordered phases and allaying our initial concern about friction effects inducing a "polar bias" in aligning collisions of elongated objects in experiments. In the metric case, the direct polar/nematic transition has been found discontinuous, in line with the order/disorder transitions, whereas the metric-free version exhibits an Ising-class continuous transition, as in equilibrium. We have signalled that a simple derivation of a continuous theory able to account for all observed facts is not easy, and constitutes an important future step for putting these results on firmer ground.

We thank Eric Bertin and Anton Peshkov for useful discussions. This work was largely performed within the activities of the Advanced Study Group "Statistical Physics of Collective Motion" at the Max Plank Institute for the Physics of Complex Systems, Dresden, Germany. FG acknowledges support by Grant IIT-Seed Artswarm.

[1] D.J. Sumpter, Collective Animal Behavior (Princeton University Press, Princeton, US, 2010)

[2] S. Ramaswamy, Annu. Rev. Condens. Matter Phys. 1, 323 (2010).

[3] P. Romanczuk et al., Eur. Phys. J. Special-Topics 202, 1 (2012).
[4] I. Giardina, HFSP J. 2, 205 (2008).

[5] T. Vicsek et al., Phys. Rev. Lett. 75, 1226 (1995).

[6] J. Toner and Y. Tu, Phys. Rev. Lett. 75, 4326 (1995); Phys. Rev. E 58, 4828 (1998); J. Toner, Phys. Rev. Lett. 108, 088102 (2012); preprint arXiv:1204.4527 (2012).

[7] G. Grégoire and H. Chaté, Phys. Rev. Lett. 92, 025702 (2004); H. Chaté, et al., Phys. Rev. E 77, 046113 (2008).

[8] F. Ginelli, F. Peruani, M. Bär, and H. Chaté, Phys. Rev. Lett. 104, 184502 (2010).

[9] For a recent work, see: F.C. Poderoso, J.J. Arenzon, and Y. Levin, Phys. Rev. Lett. 106, 067202 (2011). See also D.H. Lee and G. Grinstein, Phys. Rev. Lett. 55, 541 (1985); S. E. Korshunov, JETP 41, 263 (1985); D.B. Carpenter and J.T. Chalker, J. Phys. Condens. Matter 1, 4907 (1989).

[10] We do not expect significant changes for different densities, but a full exploration of parameter space remains to be done.

[11] There is a factor 2 between the noise strength definition used here and that used in [7], where this threshold was found near 0.2 .

[12] We cannot exclude that these two lines do not meet at the same point of the line delimiting polar order. In such a case, there would exist three different ways of crossing directly from polar to nematic order. Much longer simulations on larger systems are necessary to elucidate this.

[13] K. Binder, Rep. Prog. Phys. 60, 487 (1997); C. Borgs and R. Kotecky, J. Stat. Phys. 61, 79 (1990); Finite size scaling and numerical simulations of statistical systems, edited by V. Privman (World Scientific, Singapore, 1990).

[14] M. Moussaïd, D. Helbing, and G. Theraulaz, Proc. Natl. Acad. Sci. USA 108, 6884 (2011).

[15] M. Ballerini, et al. Proc. Natl. Acad. Sci. USA 105, 1232 (2008); W. Bialek, et al., Proc. Natl. Acad. Sci. USA 109, 4786 (2012).

[16] J. Gautrais, et al., preprint submitted to PLoS Comp. Biol. (2012).

[17] F. Ginelli, and H. Chaté, Phys. Rev. Lett. 105, 168103 (2010).

[18] A. Peshkov, et al., preprint arXiv:1203.6853 (2012).

[19] A. Gopinath, M. F. Hagan, M. C. Marchetti, and A. Baskaran, preprint arXiv:1112.6011 (2011).

[20] A. Baskaran, and M. C. Marchetti, Phys. Rev. E 77, 011920 (2008); Phys. Rev. Lett. 101, 268101 (2008); preprint arXiv:1204.3273 (2012).

[21] E. Bertin, M. Droz, and G. Grégoire, Phys. Rev. E 74, 022101 (2006); J. Phys. A 42, 445001 (2009).

[22] A more quantitative but more complicated approach, using an Enskog expansion, is able to deal directly with the Vicsek model: T. Ihle, Phys. Rev. E 83, 030901 (2011). See also Y-L Chou, R. Wolfe, and T. Ihle, preprint arXiv:1205.0830 (2012).

[23] A. Peshkov, et al., in preparation. 